\title{
Discontinuous Galerkin scheme for solving lumped kinetic model of non-isothermal liquid chromatography with Bi-Langmuir isotherms
}

\author{
Ambreen Khan, ${ }^{\dagger} \ddagger$ Sadia Perveen, ${ }^{*, \dagger}$ and Shamsul Qamar $^{\dagger}$ \\ †Department of Mathematics, COMSATS University, Park road Islamabad, 45550, \\ Pakistan \\ $\ddagger$ Department of Mathematics, Air University, PAF Complex, Sector E-9 Islamabad, 44230, \\ Pakistan \\ E-mail: *sadia.ahsan@mail.au.edu.pk
}

Appendix A: Implementations of Discontinuous Galerkin

\section{Scheme}

For a single solute elution (i.e. for $N_{c}=1$ ), the non-isothermal LKM (c.f Eqs. 10 \& 11) takes the form

$$
\begin{aligned}
\frac{\partial c_{1}}{\partial \tau}+\frac{\partial c_{1}}{\partial x}-\frac{1}{P e_{c}} \frac{\partial^{2} c_{1}}{\partial x^{2}}+F \frac{\partial q_{1}}{\partial \tau} & =0 \\
\frac{\partial c_{2}}{\partial \tau}+\frac{\partial c_{2}}{\partial x}-\frac{1}{P e_{T}} \frac{\partial^{2} c_{2}}{\partial x^{2}}+F H_{L}\left(c_{2}-q_{2}\right) & =0 \\
\frac{\partial q_{1}}{\partial \tau}-\kappa_{1}\left(q_{1}^{*}-q_{1}\right) & =0 \\
\frac{\partial q_{2}}{\partial \tau}+\frac{\Delta H_{A, 1}}{c_{e}} \frac{\partial q_{1}}{\partial \tau}-H_{S}\left(c_{2}-q_{2}\right) & =0
\end{aligned}
$$


Let us define the following two fluxes:

$$
g_{i}\left(c_{i}\right)=\frac{1}{\sqrt{P e_{i}}} \frac{\partial c_{i}}{\partial x}, \quad f_{i}\left(c_{i}, g_{i}\right)=c_{i}-\frac{1}{\sqrt{P e_{i}}} g_{i}, \quad i=1,2 .
$$

Then, PDEs in Eqs. (A-1)-(A-4) achieve the following forms:

$$
\begin{aligned}
\frac{\partial c_{1}}{\partial \tau}+\frac{\partial f_{1}}{\partial x}+F \kappa_{1}\left(q_{1}^{*}-q_{1}\right) & =0 \\
\frac{\partial c_{2}}{\partial \tau}+\frac{\partial f_{2}}{\partial x}+F H_{L}\left(c_{2}-q_{2}\right) & =0 \\
g_{i}=\frac{1}{\sqrt{P e_{i}}} \frac{\partial c_{i}}{\partial x}, \quad i & =1,2, \\
\frac{\partial q_{1}}{\partial \tau}-\kappa_{1}\left(q_{1}^{*}-q_{1}\right) & =0 \\
\frac{\partial q_{2}}{\partial \tau}+\frac{\Delta H_{A, 1}}{c_{e}} \kappa_{1}\left(q_{1}^{*}-q_{1}\right)-H_{S}\left(c_{2}-q_{2}\right) & =0
\end{aligned}
$$

If there are significant variations in the field variables across the cell interfaces (e.g. $c_{i}(x, \tau)$ in our case), the continuous finite element method is not applicable, as the implementation of continuity assumption across the cell interfaces can result in significant numerical disruptions. ${ }^{1}$ On the other hand, the important aspect behind the implementation of discontinuous Galerkin (DG) finite element method is to allow finite jump discontinuities in the field variables at the cell interfaces (c.f. Figure.S1). For solving the current model equations the DG scheme is more suitable as it provides stable solutions that are free from numerical oscillations.

To derive the DG scheme, firstly the discretization of the spatial domain $(x \in[0,1])$ is done. The partitions of the whole domain $[0,1]$ is represented by the set $\left\{x_{j+\frac{1}{2}}\right\}_{j=0}^{N}$, $I_{j}=\left(x_{j-\frac{1}{2}}, x_{j+\frac{1}{2}}\right)$ describes the $j^{\text {th }}$ cell of width $\Delta x_{j}=x_{j+\frac{1}{2}}-x_{j-\frac{1}{2}}$, and let $h=\sup _{j}\left\{\Delta x_{j}\right\}$ (c.f. Figure S1). We are looking for an approximate solution $\left(c_{i}(x, \tau)\right)_{h}$ to $c_{i}(x, \tau)$ such 


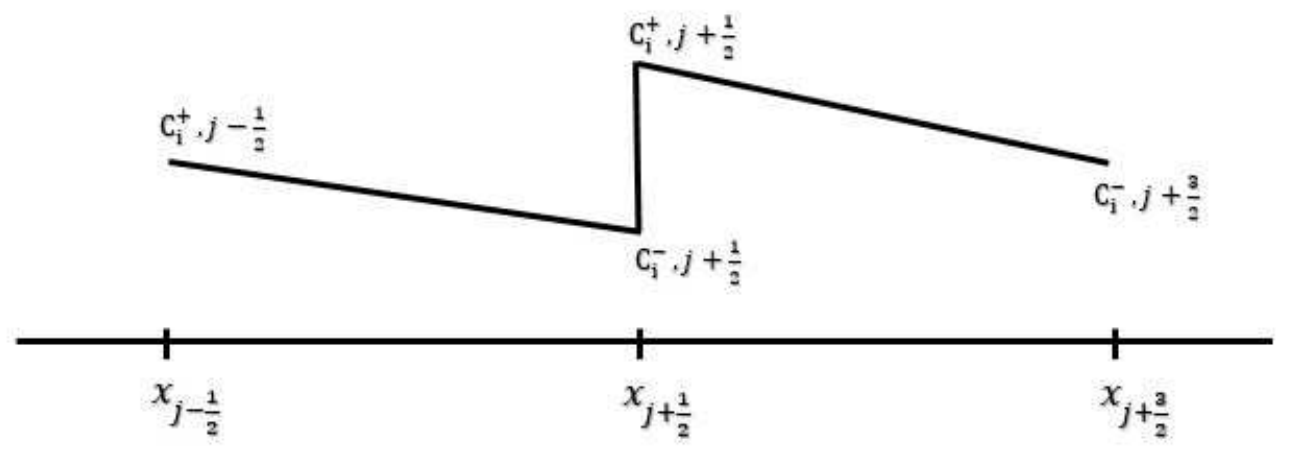

Figure S1: Illustration of jump discontinuities at the cell interfaces of the domain.

that for each $\tau \in\left[0, \tau_{\max }\right],\left(c_{i}(x, \tau)\right)_{h}$ belongs to the finite dimensional space

$$
\Phi_{h}=\left\{\phi \in L^{j}(I):\left.\phi\right|_{I_{j}} \in P_{M}\left(I_{j}\right), \quad j=1,2, \cdots, N\right\}
$$

In the space $\Phi_{h}$, functions are allowed to have jumped at the cell interfaces $x_{j \pm \frac{1}{2}}$ and $P_{M}\left(I_{j}\right)$ signify the set of polynomials of degree less than or equal to $M$ and are defined on the domain of the cell $I_{j}$ and $\tau_{\max }=u t_{\max } / L$ with $t_{\max }$ being the final simulation time. Weak formulations of the model equations are required to obtain the approximate solution $\left(c_{i}(x, \tau)\right)_{h}$ of the current model by implementing DG scheme. Eqs. (A-6)-(A-10) is multiplied with an arbitrary smooth test function $\phi(x)$ and using integration by parts over the 
interval $I_{j}$. These equations take the form as mentioned below

$$
\begin{aligned}
\int_{I_{j}} \frac{\partial c_{1}}{\partial \tau} \phi(x) d x & =-\Delta_{j+} f_{1}\left(c_{1, j-\frac{1}{2}}, g_{1, j-\frac{1}{2}}\right) \phi\left(x_{j-\frac{1}{2}}\right)+\int_{I_{j}}\left(f_{1} \frac{\partial \phi}{\partial x}-F \kappa_{1}\left(q_{1}^{*}-q_{1}\right) \phi(x)\right) d x \\
\int_{I_{j}} \frac{\partial c_{2}}{\partial \tau} \phi(x) d x & =-\Delta_{j+} f_{2}\left(c_{2, j-\frac{1}{2}}, g_{2, j-\frac{1}{2}}\right) \phi\left(x_{j-\frac{1}{2}}\right)+\int_{I_{j}}\left(f_{2} \frac{\partial \phi}{\partial x}-F H_{L}\left(c_{2}-q_{2}\right) \phi(x)\right) d x \\
\int_{I_{j}} g_{i} \phi(x) d x & =\frac{1}{\sqrt{P e_{i}}}\left\{\Delta_{j+} f_{i}\left(c_{i, j-\frac{1}{2}}, 0\right) \phi\left(x_{j-\frac{1}{2}}\right)-\int_{I_{j}} f_{i}\left(c_{i}, 0\right) \frac{\partial \phi}{\partial x} d x\right\}, i=1,2 \\
\int_{I_{j}} \frac{\partial q_{1}}{\partial \tau} \phi(x) d x & =\int_{I_{j}} \kappa_{1}\left(q_{1}^{*}-q_{1}\right) \phi(x) d x \\
\int_{I_{j}} \frac{\partial q_{2}}{\partial \tau} \phi(x) d x & =\frac{-\Delta H_{A, 1} \kappa_{1}}{c_{e}} \int_{I_{j}}\left(q_{1}^{*}-q_{1}\right) \phi(x) d x+H_{S} \int_{I_{j}}\left(c_{2}-q_{2}\right) \phi(x) d x
\end{aligned}
$$

where, $\Delta_{j+} w_{i, j-\frac{1}{2}}=w_{i, j+\frac{1}{2}}-w_{i, j-\frac{1}{2}}$. Another way to enforce Eq. (A-11) Legendre polynomials $P_{M}(x)$ that have order $M$ is to select and define them as local orthogonal basis functions over the domain element $I_{j}$. Also, the $L^{2}$-orthogonality property of Legendre polynomials can be used, that is

$$
\int_{-1}^{1} P_{n}(s) P_{n^{\prime}}(s) d s=\frac{2}{2 n+1} \delta_{n n^{\prime}}
$$

For each $x \in I_{j}$ these approximate solutions $\left(c_{i, j}\right)_{h},\left(g_{i, j}\right)_{h}$, and $\left(q_{i, j}\right)_{h}$ which are belonging to the finite dimensional space $\Phi_{h}$ can be written as linear combinations of the specifed basis functions as defined below:

$$
\left(c_{i, j}\right)_{h}=\sum_{l=0}^{M} c_{i, j}^{(l)} \phi_{l}(x), \quad\left(g_{i, j}\right)_{h}=\sum_{l=0}^{M} G_{i, j}^{(l)} \phi_{l}(x), \quad\left(q_{i, j}\right)_{h}=\sum_{l=0}^{M} q_{i, j}^{(l)} \phi_{l}(x)
$$

with

$$
\phi_{l}(x)=P_{l}\left(2\left(x-x_{j}\right) / \Delta x_{j}\right), l=0,1,2, \cdots, M
$$

Linear basis functions have used in this work that is taking $M=1$, and $l=0,1$. The 
following expression are attained by using Eqs. (A-17)-(A-19):

$$
w_{i, j}^{(l)}(t)=\frac{2 l+1}{\Delta x_{j}} \int_{I_{j}}\left(w_{i, j}\right)_{h}(\tau, x) \phi_{l}(x) d x, w \in\left\{c, g, q, q^{*}\right\}, i=1,2, j=1,2, \cdots, N .
$$

Now, the test functions $P_{l} \in \Phi_{h}$ can be used to replace the smooth function $\phi(x)$ and the approximate solutions $\left(c_{i}\right)_{h},\left(g_{i}\right)_{h}$ and $\left(q_{i}\right)_{h}$ can be used to replace the exact solutions $c_{i}$, $g_{i}$, and $q_{i}$ respectively. Also, the appropriately selected numerical flux functions can be used to replace the functions $f_{i}\left(c_{i, j \pm \frac{1}{2}}, g_{i, j \pm \frac{1}{2}}\right)$ which are not defined at cell interfaces $\partial I_{j}$.

$$
f_{i}\left(c_{i, j+\frac{1}{2}}, g_{i, j+\frac{1}{2}}\right) \approx h_{i, j+\frac{1}{2}}=h_{i}\left(c_{j+\frac{1}{2}}^{-}, c_{j+\frac{1}{2}}^{+}\right) .
$$

As $g_{i}:=g_{i}\left(c_{i}\right)$, for simplification purpose it can be dropped from the arguments of $h_{i}$ (cf. Eq. (A-21)).

Using the above definition, Eqs. (A-12)-(A-16) take the form of either ODEs or algebraic equations, as mentioned below:

$$
\begin{aligned}
\frac{d c_{1, j}^{(l)}}{d \tau} & =-\frac{2 l+1}{\Delta x_{j}} \Delta_{j+}\left(h_{1, j-\frac{1}{2}} \phi_{l}\left(x_{j-\frac{1}{2}}\right)\right)-F \kappa_{1}\left(q_{1, j}^{*(l)}-q_{1, j}^{(l)}\right)+\frac{2 l+1}{\Delta x_{j}} \int_{I_{j}} f_{1} \frac{d \phi_{l}}{d x} d x, \\
\frac{d c_{2, j}^{(l)}}{d \tau} & =-\frac{2 l+1}{\Delta x_{j}} \Delta_{j+}\left(h_{2, j-\frac{1}{2}} \phi_{l}\left(x_{j-\frac{1}{2}}\right)\right)-F H_{L}\left(c_{2, j}^{(l)}-q_{2, j}^{(l)}\right)+\frac{2 l+1}{\Delta x_{j}} \int_{I_{j}} f_{2} \frac{d \phi_{l}}{d x} d x, \\
g_{i, j}^{(l)} & =\frac{2 l+1}{\Delta x_{j}} \frac{1}{\sqrt{P e_{i}}}\left\{\Delta_{j+}\left(f_{i}\left(c_{i, j-\frac{1}{2}}, 0\right) \phi_{l}\left(x_{j-\frac{1}{2}}\right)\right)-\int_{I_{j}} f_{i}\left(c_{i}, 0\right) \frac{d \phi_{l}}{d x}\right\}, \quad i=1,2, \\
\frac{d q_{1, j}^{(l)}}{d \tau} & =\kappa_{1}\left(q_{1, j}^{*(l)}-q_{1, j}^{(l)}\right), \\
\frac{d q_{2, j}^{(l)}}{d \tau} & =\frac{-\Delta H_{A} \kappa_{1}}{c_{e}}\left(q_{1, j}^{*(l)}-q_{1, j}^{(l)}\right)+H_{S}\left(c_{2, j}^{(l)}-q_{2, j}^{(l)}\right),
\end{aligned}
$$

along with the initial data, which is expressed in the form of new weak formulation as 
(c.f. Eq. (A-20))

$$
\begin{aligned}
c_{i, j}^{(l)}(0) & =\frac{2 l+1}{\Delta x_{j}} \int_{I_{j}} c_{i}(x, 0) \phi_{l}(x) d x, \\
g_{i, j}^{(l)}(0) & =h\left(c_{i, j}^{(l)}(0)\right), \quad q_{i, j}^{(l)}(0)=q\left(c_{i, j}^{(l)}(0)\right), \quad i=1,2 .
\end{aligned}
$$

It is necessary to choose an accurate numerical flux function $h_{i}$ to measure the cell interface fluxes values for the effective functioning of the current numerical scheme. To achieve accurate numerical results by specifying a numerical flux function which is consistent (i.e. $\left.h_{i}\left(c_{i}, c_{i}\right)=f_{i}\left(c_{i}\right)\right)$, monotone and Lipschitz continuous which means $h_{i}(\cdot, \cdot)$ should be nondecreasing in its first argument and non-increasing in its second argument. ${ }^{2}$ Literature $^{3-5}$ provides a number of such numerical flux functions. For the proposed model, the Local Lax-Friedrich numerical flux is utilized:

$$
h_{i}^{\mathrm{LLF}}(a, b)=\frac{1}{2}\left[f_{i}\left(a, g_{i}(a)\right)+f_{i}\left(b, g_{i}(b)\right)-C(b-a)\right],
$$

where

$$
C=\max _{\min (a, b) \leq s \leq \max (a, b)}\left|f_{i}^{\prime}\left(s, g_{i}(s)\right)\right|
$$

The scheme's total variation stability can be attained through modification of $c_{i, j \pm \frac{1}{2}}^{ \pm}$by utilizing a local projection limiter. Thus

$$
\bar{c}_{i, j+\frac{1}{2}}=c_{i, j}^{(0)}+\tilde{c}_{i, j}, \quad \widetilde{\widetilde{c}}_{i, j-\frac{1}{2}}=c_{i, j}^{(0)}-\widetilde{\widetilde{c}}_{i, j}
$$


where,

$$
\tilde{c}_{i, j}=\sum_{l=1}^{M} c_{i, j}^{(l)} \phi_{l}\left(x_{j+\frac{1}{2}}\right), \quad \widetilde{\widetilde{c}}_{i, j}=-\sum_{l=1}^{M}\left(c_{i, j}\right)^{(l)} \phi_{l}\left(x_{j-\frac{1}{2}}\right) .
$$

We have considered linear basis functions $M=1$ in the current manuscript. The expressions given in Eq. (A-31) are updated to confirm the local positivity by using Van Leer slope limiters ${ }^{6,7}$ which are expressed as

$$
\tilde{c}_{i, j}=\operatorname{mm}\left(\tilde{c}_{i, j}, \Delta_{j+} c_{i, j}^{(0)}, \Delta_{j+} c_{i, j}^{(0)}\right), \quad \widetilde{\widetilde{c}}_{i, j}=\operatorname{mm}\left(\widetilde{\widetilde{c}}_{i, j}, \Delta_{j+} c_{i, j}^{(0)}, \Delta_{j+} c_{i, j}^{(0)}\right)
$$

where, the minmod function mm is defined as

$$
\operatorname{mm}\left(v_{1}, v_{2}, v_{3}\right)=\left\{\begin{array}{lc}
s \cdot \min _{1 \leq j \leq 3}\left|v_{j}\right|, & \text { if } \operatorname{sign}\left(v_{1}\right)=\operatorname{sign}\left(v_{2}\right)=\operatorname{sign}\left(v_{3}\right)=s \\
0, & \text { otherwise }
\end{array}\right.
$$

Eq. (A-30) can be written as

$$
\bar{c}_{i, j+\frac{1}{2}}^{(\mathrm{mod})}=c_{i, j}^{(0)}+\widetilde{c}_{i, j}, \quad \bar{c}_{i, j-\frac{1}{2}}^{(\bmod )}=c_{i, j}^{(0)}-\widetilde{\widetilde{c}}_{i, j}^{(\bmod )}
$$

and Eq. (A-21) is modified as

$$
h_{i, j+\frac{1}{2}}=h_{i}\left(\bar{c}_{i, j+\frac{1}{2}}^{(\mathrm{mod})}, \stackrel{+}{c}_{i, j-\frac{1}{2}}^{(\bmod )}\right)
$$

The established local projection limiter in Eqs.(A-32)-(A-34) imposes stability of the scheme without disrupting its order of accuracy. The implementation of LDG method has provided us with a system of ODEs (c.f. Eqs. (A-22)-(A-26)) that are precisely given 
below

$$
\frac{d \mathbf{w}_{h}}{d \tau}=L_{h}\left(\mathbf{w}_{h}, t\right)
$$

where, $\mathbf{w}_{h}=\left[c_{1}, c_{2}, q_{1}, q_{2}\right]^{T}$. The above system of ODEs are solved by using the $r^{\text {th }}-$ order TVB RK-method as follows

$$
\mathbf{w}_{h}^{(m)}=\sum_{l=0}^{m-1}\left\{\alpha_{m l} \mathbf{w}_{h}^{(l)}+\gamma_{m l} \Delta \tau L_{h}\left(\mathbf{w}_{h}^{(l)}, \tau^{n}+d_{l} \Delta \tau\right)\right\}, \quad m=1,2, \ldots, r
$$

where the initial data is written as (c.f. eq.(A-28))

$$
\mathbf{w}_{h}^{(0)}=\mathbf{w}_{h}^{n}, \quad \mathbf{w}_{h}^{(r)}=\mathbf{w}_{h}^{n+1},
$$

with $n$ being the time step number. The coefficients of TVB-RK method for a second scheme are expressed as ${ }^{8,9}$

$$
\alpha_{10}=\gamma_{10}=1, \quad \alpha_{20}=\alpha_{21}=\gamma_{21}=\frac{1}{2}, \quad \gamma_{20}=d_{0}=0, \quad d_{1}=1
$$

While, for a third order scheme, they are defined as

$$
\begin{array}{r}
\alpha_{10}=\gamma_{10}=1, \quad \alpha_{20}=\frac{3}{4}, \quad \gamma_{20}=0, \quad \alpha_{21}=\gamma_{21}=\frac{1}{4} \\
\alpha_{30}=\frac{1}{3}, \quad \gamma_{30}=\alpha_{31}=\gamma_{31}=0, \quad \alpha_{32}=\gamma_{32}=\frac{2}{3} \\
d_{0}=0, \quad d_{1}=1, \quad d_{2}=\frac{1}{2}
\end{array}
$$

\section{Implementation of boundary conditions:}

For the purpose of applying the boundary conditions, like the Cockburn and Shu ${ }^{2}$ we put the left end of the boundary at $x_{-\frac{1}{2}}$ (i.e. $\left.x_{-\frac{1}{2}}=0\right)$. Then the boundary conditions (c.f. Eq. 
7) for the above referred scheme is converted as

$$
\begin{aligned}
\bar{c}_{i,-\frac{1}{2}}(t) & =c_{i}(0, t), \stackrel{\sim}{c}(\bmod )_{i, 0}=\operatorname{mm}\left(\tilde{c}_{i, 0}, \Delta_{j+} c_{i, 0}^{(0)}, 2\left(c_{i, 0}^{(0)}-c_{i}(0, t)\right)\right), \\
\widetilde{\widetilde{c}}_{i, 0}^{(\mathrm{mod})} & =\operatorname{mm}\left(\widetilde{\widetilde{c}}_{i, 0}, \Delta_{j+} c_{i, 0}^{(0)}\right) .
\end{aligned}
$$

On the other end of the column outflow boundary conditions are used

$$
\left.\frac{\partial c_{i}^{(l)}}{\partial x}\right|_{x=1}=0 .
$$

In a similar pattern, the scheme is adapted for multi-component flows.

\section{Appendix B: Effects of enthalpy of adsorption}

In the following, the impact of enthalpy of adsorption are discussed for single-component, two-component and three component chromatographic elutions with Bi-Langmuir isotherm repectively.

\section{Non-Isothermal single-component chromatographic elution}

The effect of different values of $\Delta H_{A}$ on concentration and temperature profiles are shown in Figure. S2 for single-component chromatographic elution discussed in section 4.1. It is observed that the greater value of adsorption enthalpy has an important influence on the temperature and concentration profiles. The adsorption front of temperature profile becomes narrower and elutes earlier, although the height of desorption slows down and spreads. The maximum peak for the concentration profile is obtained at $\Delta H_{A}=0$ while the minimum peak for the concentration profile is achieved at $\Delta H_{A}=-60 \mathrm{~kJ} / \mathrm{mol}$ and the profile is more impacted due to changes in temperature. Also, the effect of isotherm's 
non-linearity can be seen from the steeper and asymmetrical patterns of the concentration profile for increasing values of $\Delta H_{A}$.

\section{Non-Isothermal two-component chromatographic elution}

The effects of adsorption enthalpy for two-components chromatographic elution discussed in section 4.2 are illustrated in the Figures. S3-S5. Concentration and temperature profiles

plots are produced for mass transfer rate coefficients $k=10 \mathrm{~min}^{-1}$ and $k=100 \mathrm{~min}^{-1}$. By increasing the absolute value of adsorption enthalpy, particularly in the case of $k=100$ $\min ^{-1}$, a significant separation between the two mixture components can be obtained. For both values of $k$ non-isothermal operating conditions along with the considered adsorption dynamics generate significant fluctuation in the concentration and temperature profiles. The impact of different values of $\Delta H_{A}=0,-20,-40,-60 \mathrm{KJ} / \mathrm{mol}$ with non-equal injection for the grid independence test study discussed in section 4.2 is presented in Figures. S6S7. Visible fluctuation can be observed in the concentration and temperature profiles for $\Delta H_{A}=-20 \mathrm{KJ} / \mathrm{mol}$ and $\Delta H_{A}=-40 \mathrm{KJ} / \mathrm{mol}$. For $\Delta H_{A}=0 \mathrm{KJ} / \mathrm{mol}$ and $\Delta H_{A}=-60$ $\mathrm{KJ} / \mathrm{mol}$, the RKLDG method is capable of providing non-oscillatory solution.

\section{Non-isothermal three-component chromatographic elution}

The suggested scheme is extended to non-isothermal and non-linear three-component application of LKM. In this numerical test study, mass transfer coefficients, adsorption enthalpy and the dispersion coefficient are considered to be the same for all components of the mixture. Figure. S8 shows that how the band-broadening, overlapping and the retention times of the three component elution curves are changing for different values of adsorption enthalpy. The complete set of model parameters used in the simulations are $\epsilon=0.4, u=1.0$ $\mathrm{cm} / \mathrm{min}, a_{1 \mathrm{I}}^{\mathrm{ref}}=0.5 a_{1 \mathrm{II}}^{\mathrm{ref}}=1.0, a_{2 \mathrm{I}}^{\mathrm{ref}}=0.75, a_{2 \mathrm{II}}^{\mathrm{ref}}=2.5, a_{3 \mathrm{I}}^{\mathrm{ref}}=2.5 a_{\mathrm{II}}^{\mathrm{ref}}=2.0, b_{1 \mathrm{I}}^{\mathrm{ref}}=0.25$, 
$b_{1 I I}^{\mathrm{ref}}=2.0, b_{2 \mathrm{I}}^{\mathrm{ref}}=0.25, b_{2 \mathrm{II}}^{\mathrm{ref}}=1.0, b_{3 \mathrm{I}}^{\mathrm{ref}}=0.25, b_{3 \mathrm{II}}^{\mathrm{ref}}=2.0, D_{L, i}=D_{L}=0.0001 \mathrm{~cm}^{2} / \mathrm{min}$, $k_{i}=10 \mathrm{~min}^{-1}$ and $t_{\max }=120 \mathrm{~min}$. A rectangular pulse of feed concentration of height one with $c_{\mathrm{i}, \text { inj }}=1 \mathrm{~mol} / \mathrm{l} i=1,2,3$ is injected for the duration of $t_{i n j}=5 \mathrm{~min}$ into the column of length $L=10 \mathrm{~cm}$. The resolution capability of the components, impact of temperature variations on their shape, the development of sharp fronts in the concentration profiles, and the steep front of elution profiles are more apparent with increasing value of $\Delta H_{A}$.

\section{Authours Information}

\section{Corresponding Author}

Email: *sadia.ahsan@mail.au.edu.pk

Note: The authors declare no competing financial interest.

\section{References}

(1) Zhou, J. G.; Causon, D. M.; Mingham, C. G.; Ingram, D. M. The surface gradient method for the treatment of source terms in the shallow-water equations. J. Comput. Phys. 2001, 168(1), 1-25.

(2) Cockburn, B.; Shu, C. W. TVB Runge-Kutta local projection discontinuous Galerkin finite element method for conservation laws. II. General framework. Math. Comput. 1989, 52(186), 411-435.

(3) Zhang, P.; Liu, R. X. Hyperbolic conservation laws with space-dependent fluxes: II. General study of numerical fluxes. Journal of Computational and Applied Mathematics 2005, 176(1), 105-129. 
(4) LeVeque, R. J. Finite volume methods for hyperbolic problems (Vol. 31). Cambridge university press. 2002

(5) Kurganov, A.; Tadmor, E. New high-resolution central schemes for nonlinear conservation laws and convection-diffusion equations. J. Comput. Phys . 2000, 160(1), $241-282$.

(6) Jiang, G. S.; Shu, C. W. On a cell entropy inequality for discontinuous Galerkin methods. Mathematics of Computation 1994, 62(206), 531-538.

(7) Johnson, C. Error estimates and adaptive time-step control for a class of one-step methods for stiff ordinary differential equations. SIAM J. Numer. Anal. 1988, 25(4), 908-926.

(8) Cockburn, B.; Shu, C. W. Runge-Kutta discontinuous Galerkin methods for convection-dominated problems. Journal of scientific computing 2001, 16(3), 173261.

(9) Javeed, S.; Qamar, S.; Seidel-Morgenstern, A.; Warnecke, G. A discontinuous Galerkin method to solve chromatographic models. J. Chromatogr. A. 2016, $1218(40), 7137-7146$. 

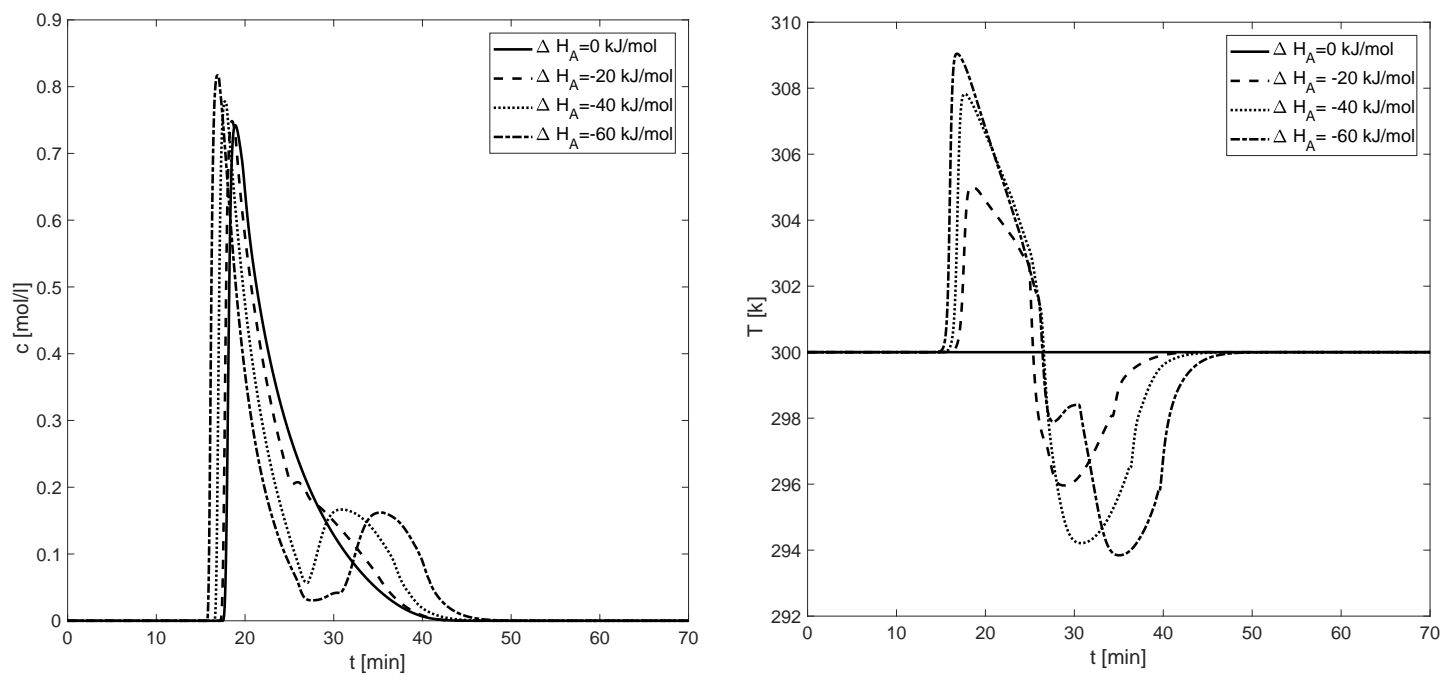

Figure S2: Effect of $\Delta H_{A}$ on concentration and temperature profiles. 

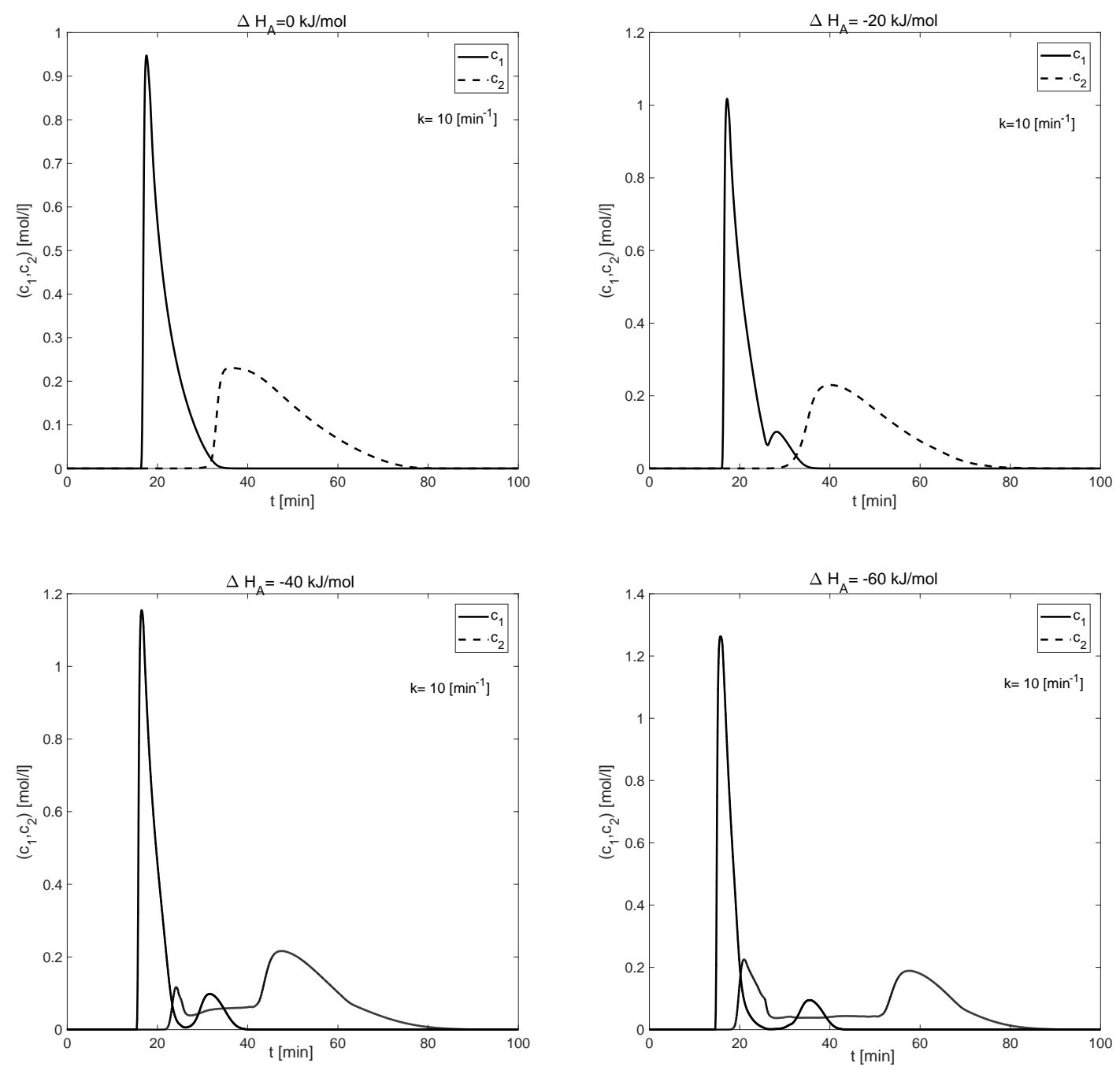

Figure S3: Effect of $\Delta H_{A}$ on separation of concentration profiles in the case of two component mixture for $k=10 \mathrm{~min}^{-1}$. 

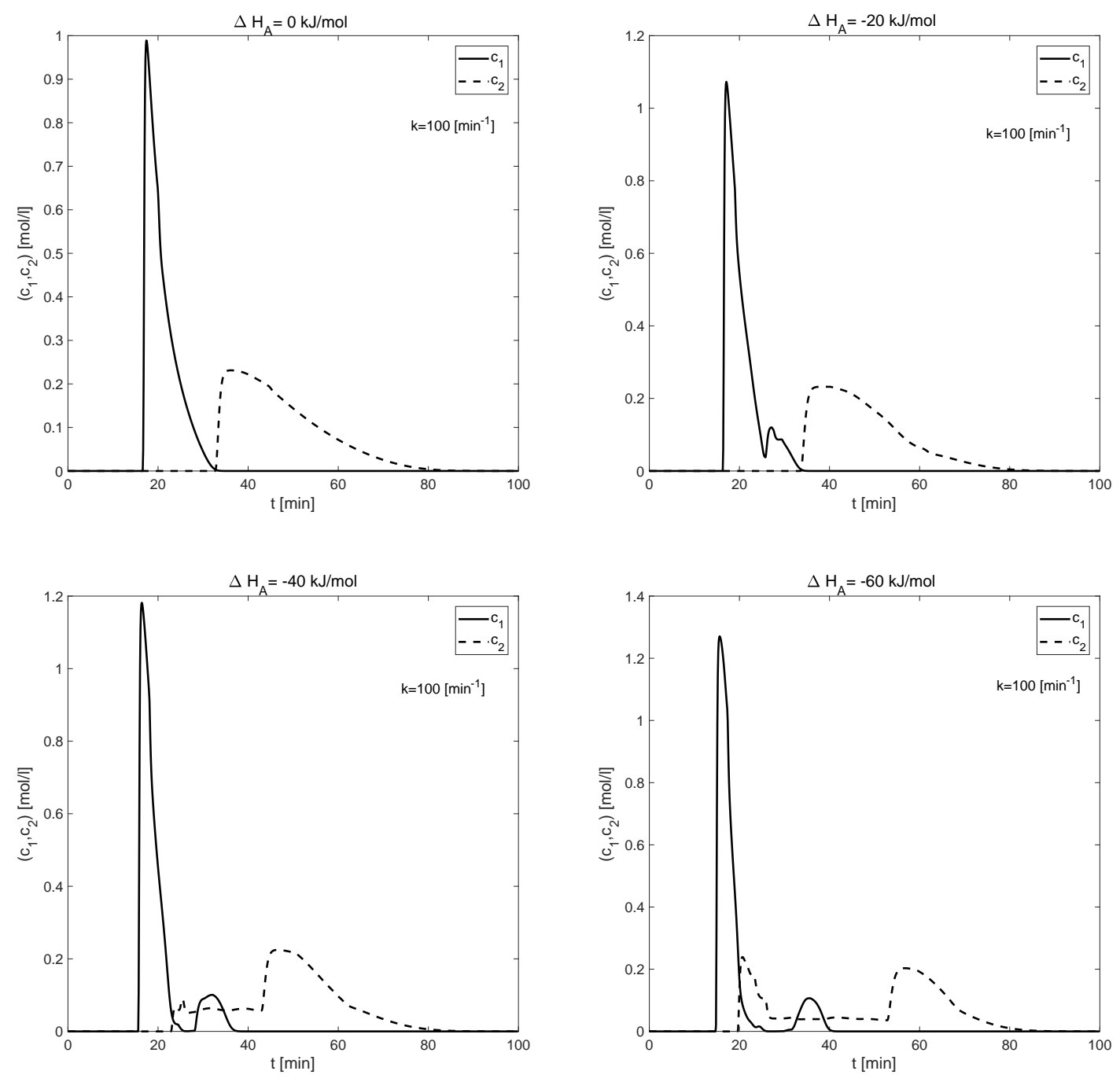

Figure S4: Effect of $\Delta H_{A}$ on the separation of concentration profiles in the case of two component mixture for $k=100 \mathrm{~min}^{-1}$. 

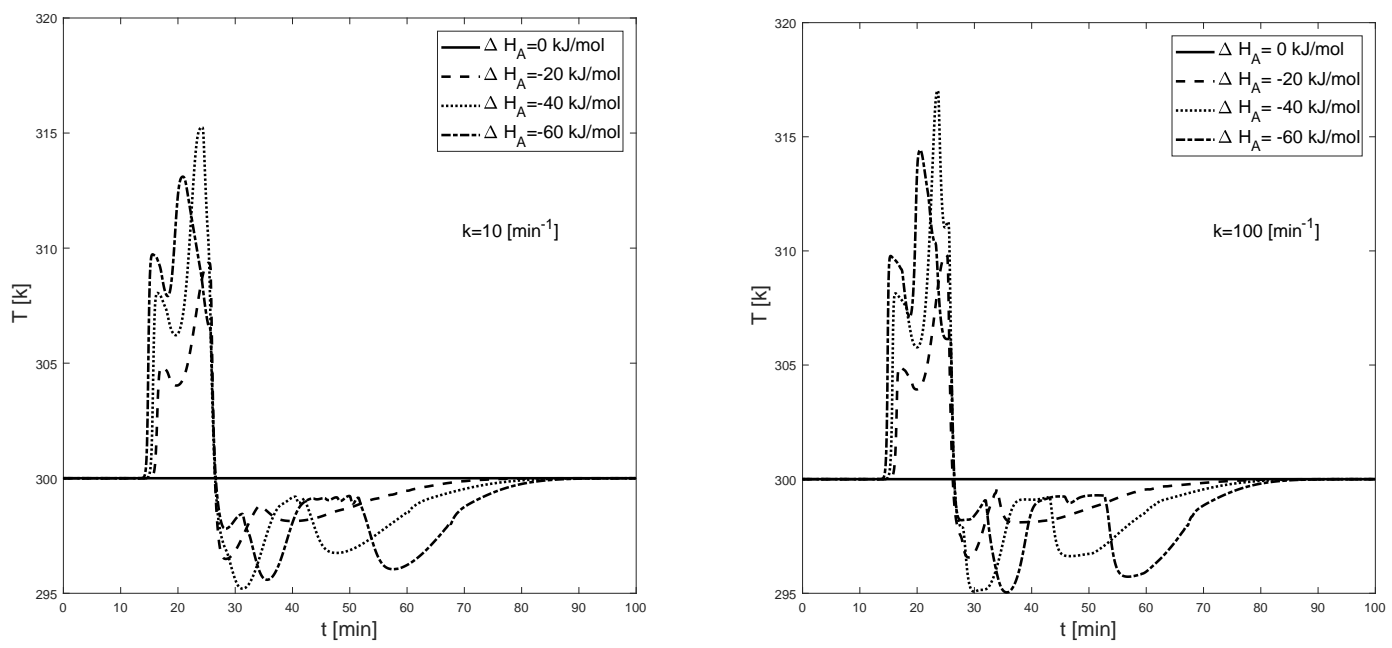

Figure S5: Effect of $\Delta H_{A}$ on the temperature profiles of two component mixture for the cases $k=10 \mathrm{~min}^{-1}$ and $k=100 \mathrm{~min}^{-1}$. 

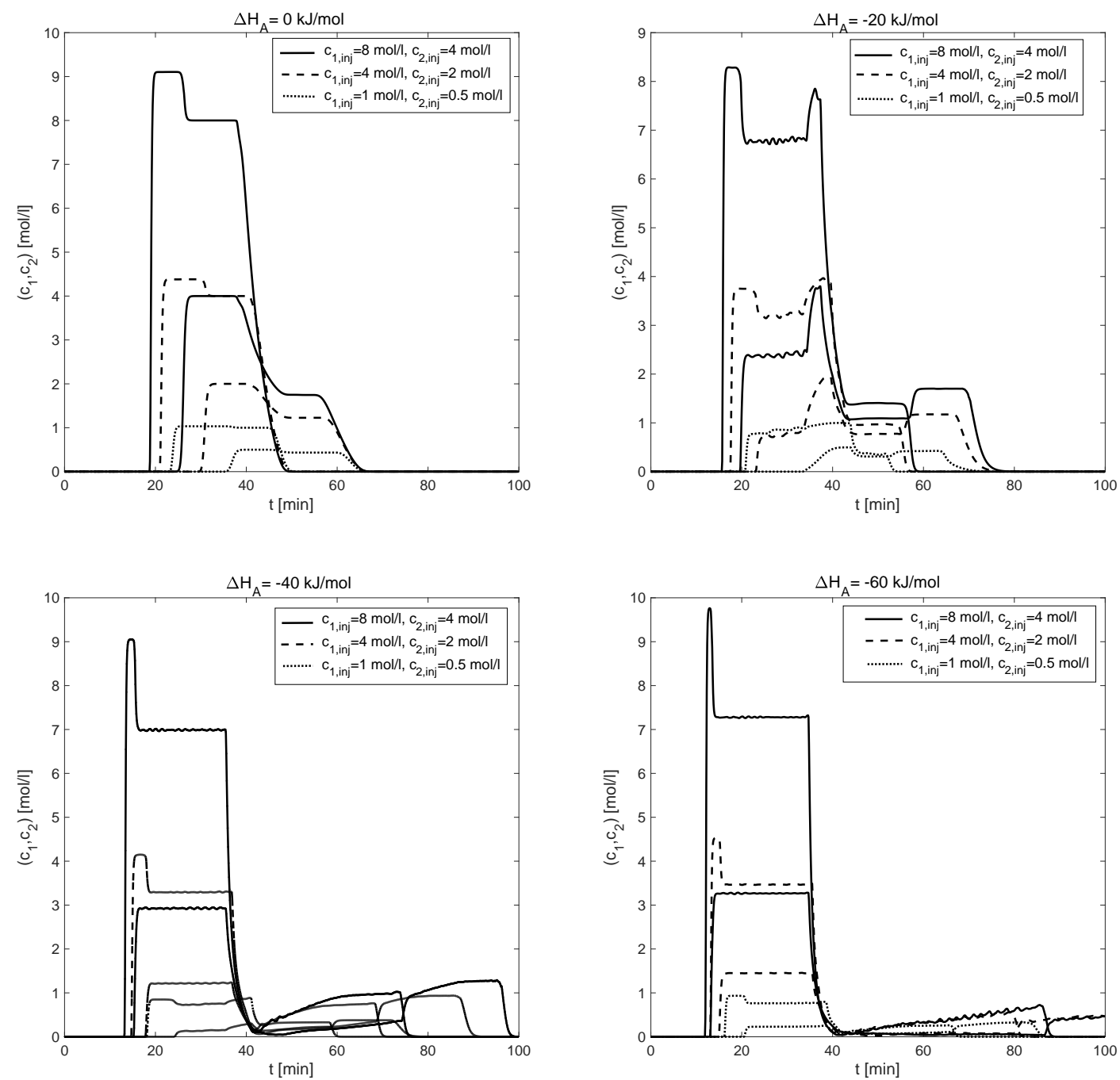

Figure S6: Effect of $\Delta H_{A}$ on the concentration profiles for three different injection volumes is shown for $k=100 \mathrm{~min}^{-1}$. The first injection: $c_{1, \text { inj }}=4 \mathrm{~mol} / 1 \& c_{2, \text { inj }}=2 \mathrm{~mol} / \mathrm{l}$, second injection: $c_{1, \mathrm{inj}}=2 \mathrm{~mol} / 1 \& c_{2, \mathrm{inj}}=1 \mathrm{~mol} / \mathrm{l}$, and the third injection: $c_{1, \mathrm{inj}}=1 \mathrm{~mol} / \mathrm{l} \&$ $c_{2, \text { inj }}=0.5 \mathrm{~mol} / \mathrm{l}$. 

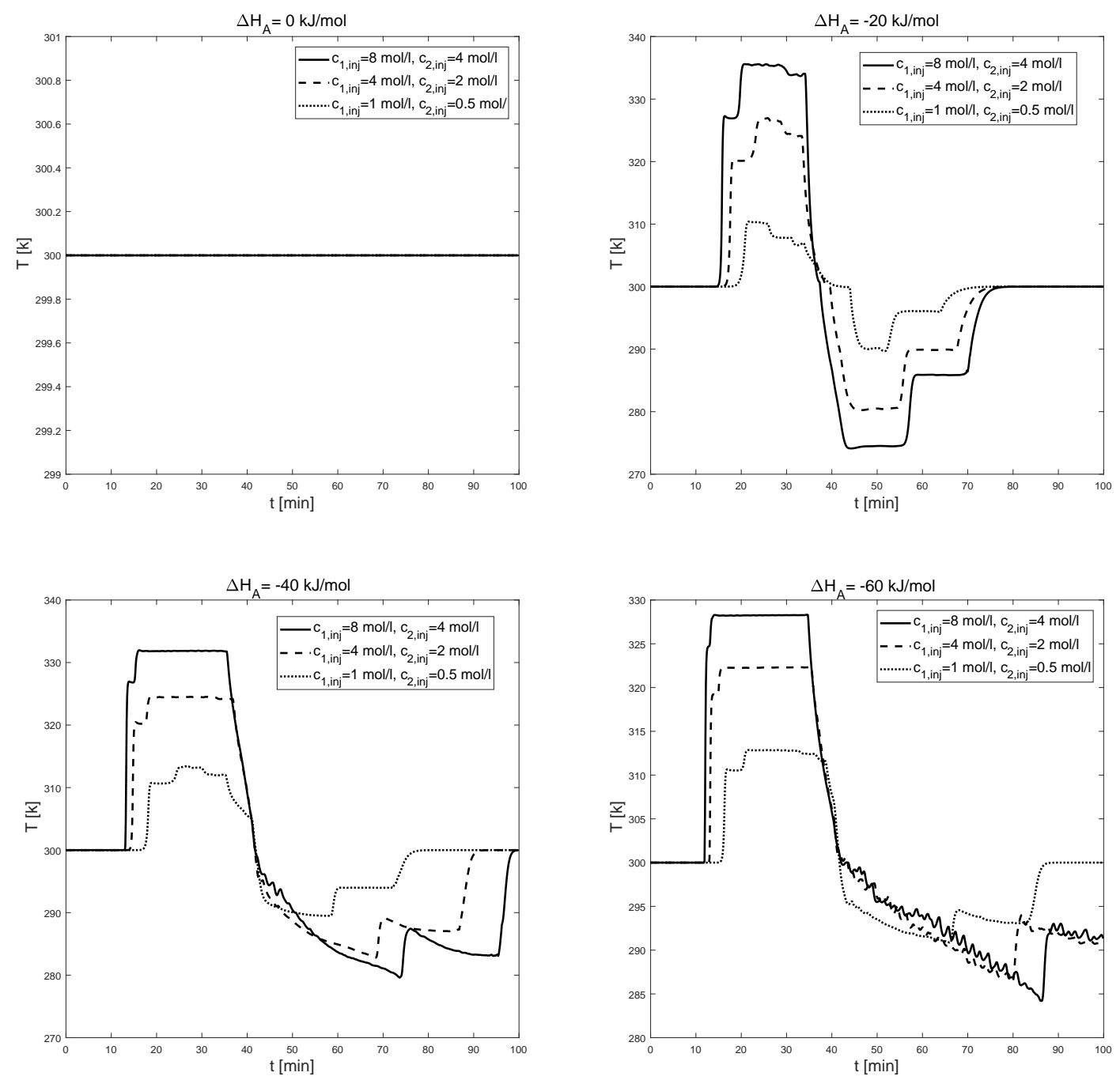

Figure S7: Effect of $\Delta H_{A}$ on the temperature profiles for different injection volumes is shown for $k=100 \mathrm{~min}^{-1}$. The first injection: $c_{1, \text { inj }}=4 \mathrm{~mol} / 1 \& c_{2, \text { inj }}=2 \mathrm{~mol} / \mathrm{l}$, the second injection: $c_{1, \text { inj }}=2 \mathrm{~mol} / \mathrm{l} \& c_{2, \mathrm{inj}}=1 \mathrm{~mol} / \mathrm{l}$, and the third injection: $c_{1, \mathrm{inj}}=1 \mathrm{~mol} / \mathrm{l} \&$ $c_{2, \text { inj }}=0.5 \mathrm{~mol} / \mathrm{l}$. 

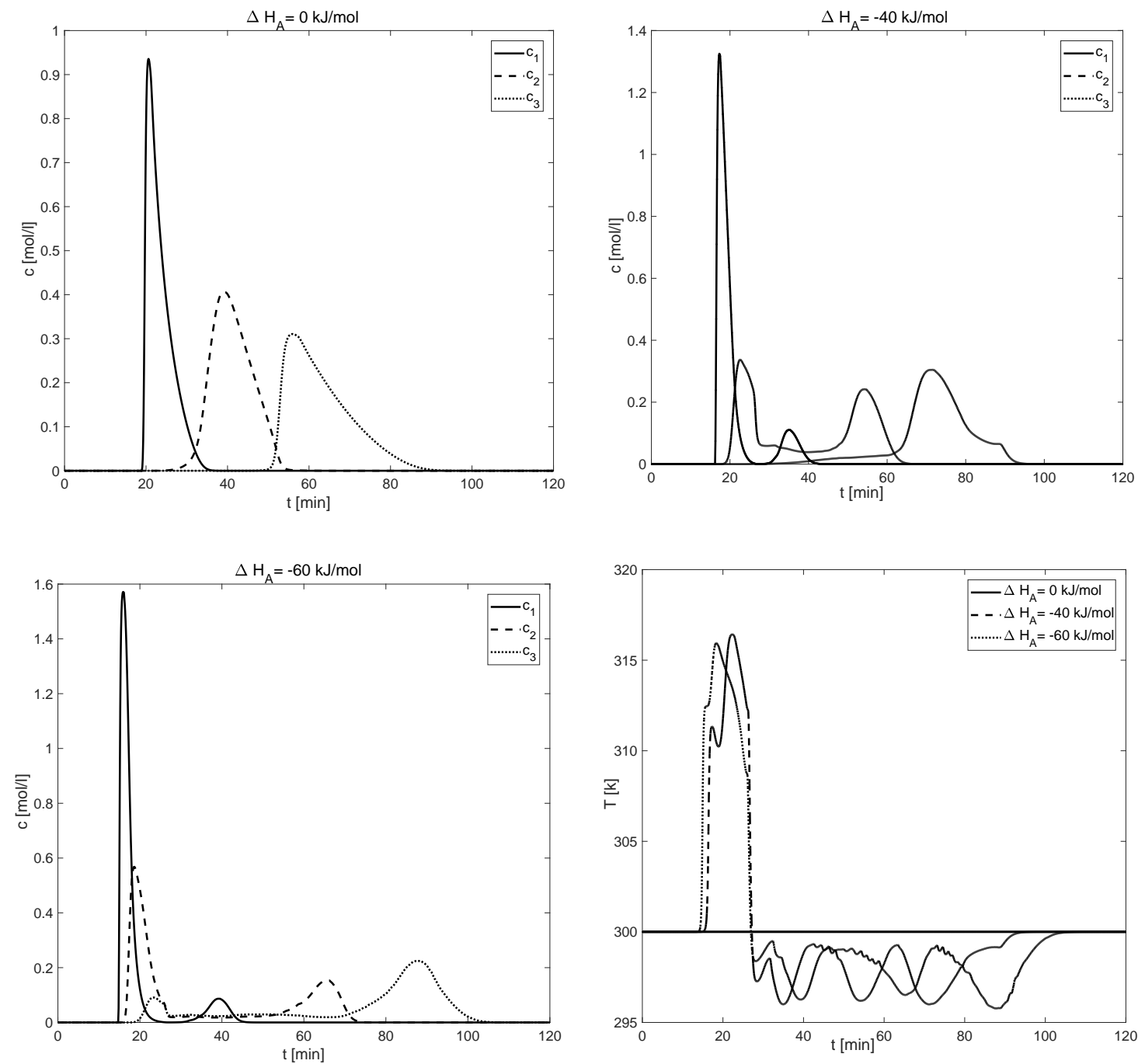

Figure S8: Effect of $\Delta H_{A}$ on three component mixture separation by using RKLDG method and $k=10 \mathrm{~min}^{-1}$. 